Revue internationale P.M.E.

Économie et gestion de la petite et moyenne entreprise

\title{
La finance corporative comme objet d'étude dans la littérature scientifique sur la PME
}

\section{Claude Francoeur}

Volume 14, numéro 1, 2001

URI : https://id.erudit.org/iderudit/1008688ar

DOI : https://doi.org/10.7202/1008688ar

Aller au sommaire du numéro

Éditeur(s)

Presses de l’Université du Québec

ISSN

0776-5436 (imprimé)

1918-9699 (numérique)

Découvrir la revue

Citer cette note

Francoeur, C. (2001). La finance corporative comme objet d'étude dans la littérature scientifique sur la PME. Revue internationale P.M.E., 14(1), 93-111. https://doi.org/10.7202/1008688ar

\section{Résumé de l'article}

Cette étude vise à déterminer les caractéristiques des objets de recherche en finance corporative des PME. La recherche porte sur 108 articles publiés durant les 10 dernières années dans six revues scientifiques de renom dans le domaine de l'entrepreneuriat et de la gestion des PME : Entrepreneurship Theory and Practice, International Small Business Journal, Journal of Business Venturing, Journal of Small Business Management, Revue internationale PME et Small Business Economics. Tous les objets de recherche ont été identifiés à partir du texte intégral des articles recueillis et classés a posteriori dans l'une ou l'autre des catégories suivantes : le capital de risque, la création d'entreprises, les conditions de financement, l'entre-preneurship, l'évaluation, la gestion financière, l'objectif financier, la performance financière, la recherche, la structure de capital et la structure organisationnelle. Ces objets de recherche ont aussi été analysés en fonction des recommandations de Pettit et Singer (1985) sur ce que devraient être les voies de recherche à emprunter en finance corporative des PME ; les écarts entre les pistes de recherche suggérées et les objets effectivement étudiés sont analysés et discutés. Les résultats font notamment ressortir la grande diversité des auteurs, une forte concentration autour d'un nombre restreint d'objets de recherche et un manque d'intérêt pour certaines autres voies de recherche jugées, par ailleurs, importantes pour le développement des connaissances en finance des PME.
Ce document est protégé par la loi sur le droit d'auteur. L'utilisation des services d’Érudit (y compris la reproduction) est assujettie à sa politique d'utilisation que vous pouvez consulter en ligne.

https://apropos.erudit.org/fr/usagers/politique-dutilisation/ 


\title{
La finance corporative comme objet d'étude dans la littérature scientifique sur la PME
}

\author{
Claude FRANCOEUR \\ Université du Québec à Montréal
}

\author{
MOTS CLÉS
}

\section{Finance corporative - Recherche - Connaissances Épistémologie - Méta-analyse}

\begin{abstract}
RÉSUMÉ
Cette étude vise à déterminer les caractéristiques des objets de recherche en finance corporative des PME. La recherche porte sur 108 articles publiés durant les 10 dernières années dans six revues scientifiques de renom dans le domaine de l'entrepreneuriat et de la gestion des PME : Entrepreneurship Theory and Practice, International Small Business Journal, Journal of Business Venturing, Journal of Small Business Management, Revue internationale PME et Small Business Economics. Tous les objets de recherche ont été identifiés à partir du texte intégral des articles recueillis et classés a posteriori dans l'une ou l'autre des catégories suivantes: le capital de risque, la création d'entreprises, les conditions de financement, l'entrepreneurship, l'évaluation, la gestion financière, l'objectif financier, la performance
\end{abstract}

\section{L'AUTEUR}

Claude Francoevr est candidat au doctorat en finance (Ph. D.) à l'École des sciences de la gestion de l'Université du Québec à Montréal. II détient une maîtrise en administration des affaires (MBA) et un titre comptable professionnel (CGA). Il a œuvré pendant plusieurs années comme administrateur dans un grand centre universitaire de recherche médicale. Ses intérêts actuels de recherche portent sur divers aspects de la finance corporative, notamment le caractère spécifique des PME, l'évaluation de la firme, l'analyse des fusions et des acquisitions et la prévision de la performance. Adresse: Département stratégie des affaires, École des sciences de la gestion, Université du Québec à Montréal, case postale 6192, succursale Centre-ville, Montréal, Québec, H3C 4R2.

L'auteur remercie Pierre Cossette pour ses judicieux commentaires lors de la préparation de ce document. 
financière, la recherche, la structure de capital et la structure organisationnelle. Ces objets de recherche ont aussi été analysés en fonction des recommandations de Pettit et Singer (1985) sur ce que devraient être les voies de recherche à emprunter en finance corporative des PME; les écarts entre les pistes de recherche suggérées et les objets effectivement étudiés sont analysés et discutés. Les résultats font notamment ressortir la grande diversité des auteurs, une forte concentration autour d'un nombre restreint d'objets de recherche et un manque d'intérêt pour certaines autres voies de recherche jugées, par ailleurs, importantes pour le développement des connaissances en finance des PME.

\begin{abstract}
The purpose of this study is to determine the characteristics of the topics of study in SME's corporate finance. This research is based on 108 papers published in the last 10 years in six prestigious scientific journals in the field of entrepreneurship and SME management: Entrepreneurship Theory and Practice, International Small Business Journal, Journal of Business Venturing, Journal of Small Business Management, Revue internationale PME and Small Business Economics. The integral text of the retained articles is the basis to identify its topic of research which is classified a posteriori in one of the following categories : venture capital, business start-up, financing conditions, entrepreneurship, valuation, financial management, financial objective, financial performance, research, capital structure and organizational structure. These topics of research are also analyzed relative to Pettit and Singer's (1985) recommendations as to what should be the research avenues to be followed in SME's corporate finance; the gaps between the suggested research paths and the topics that were effectively studied are analyzed and discussed. The results show clearly the great diversity of authors, a strong concentration around a small number of research topics and a lack of interest for other avenues of research that are otherwise considered important for the development of knowledge in SME's finance.
\end{abstract}

\title{
RESUMEN
}

Este estudio tiene como objetivo determinar las características de los tópicos de investigación en finanzas corporativa de las PyMEs. La investigación se basa en 108 artículos publicados, durante los últimos 10 años, en 6 revistas científicas reconocidas en el tópico del empresariado y de la administración de las PyMEs: Entrepreneurship Theory and Practice, International Small Business Journal, Journal of Business Venturing, Journal of Small Business Management, Revue internationale PME y Small Business Economics. Todos los tópicos de estudios son identificados a partir del texto integral de los articulos recogidos y clasificados a posteriori en una u otra de las categorías siguientes : capital de riesgo, creación de empresas, condiciones de financiamiento, espíritu empresarial, evaluación, administración financiera, objetivo financiero, rendimiento financiera, investigación, estructura de capital y estructura organizacional. Estos tópicos de investigación son también analizados en función de las recomendaciones de Pettit et Singer (1985) sobre lo que tendrían que ser las vías de investigación basadas en las finanzas corporativas de las PyMEs ; las brechas entre las pistas de investigación sugeridas y los tópicos efectivamente estudiados son analizadas y discutidas. Los resultos hacen resaltar la grande diversidad de los autores, una fuerte concentración alrededor de un 
numero pequeño de tópicos de investigación y una falta de interés por ciertas otras vías de investigación juzgadas, por otro lado importantes para el desarrollo de los conocimientos en finanzas de las PyMEs.

\section{ZUSAMMENFASSUNG}

Diese Untersuchung hat sich zum Ziel gesetzt, die charakteristischen Merkmale von Finanzgenossenschaften bei kleinen und mittleren Unternehmungen zu bestimmen. Die Untersuchung basiert auf 108 publizierten Artikeln, die während den letzten 10 Jahren in sechs renommierten, wissenschaftlichen Zeitschriften im Bereich des Unternehmertums und der Führung von kleinen und mittleren Unternehmen veröffentlicht wurden: Entrepreneurship Theory and Practice, International Small Business Journal, Journal of Business Venturing, Journal of Small Business Management, Revue internationale PME und Small Business Economics.

Alle Untersuchungsobjekte wurden unverkürzt aufgenommen und in folgende Kategorien klassiert: Risikokapital, Unternehmungsgründung, Finanzierungsbedingungen, Unternehmertum, Bewertung, Finanzielle Führung, Finanzielle Leistung, Forschung, Kapitalstruktur und organisationelle Struktur. Im weiteren wurden in Anlehnung an Pettit et Singer (1995) die Untersuchungsobjekte analysiert. Die Unterschiede zwischen den vorerst wahrgenommenen Forschungswegen und den effektiv studierten Untersuchungsobjekten wurden aufgezeigt und diskutiert.

\section{Introduction}

Les connaissances actuelles en matière de finance corporative ont été acquises durant les 50 dernières années surtout à partir de recherches effectuées auprès de grandes entreprises. Par ailleurs, on reconnaît généralement que la recherche sur la PME connaît un essor considérable depuis quelques années (voir, notamment, Cossette, 1995). Plus spécifiquement, McMahon et Stanger (1995) affirment que la recherche sur la finance corporative des PME attire de plus en plus l'attention de la communauté scientifique ces dernières années. En revanche, selon Brophy et Shulman (1992), très peu de chercheurs ont tenté d'adapter les modèles existants ou de développer de nouvelles théories financières qui tiennent compte des particularités des PME. Malgré l'engouement relatif pour la recherche en entrepreneuriat, il n'existe toujours pas de base théorique reconnue qui tienne compte de la spécificité des PME (Colot et Michel, 1996).

La présente étude vise à faire état des types de recherches effectuées en finance corporative des PME durant les 10 dernières années, à en dégager les caractéristiques et à en analyser l'évolution. Après avoir présenté le cadre opératoire dans lequel notre étude s'insère et exposé les résultats obtenus, nous en effectuerons l'analyse et en dégagerons les principales caractéristiques. De plus, les objets de recherche répertoriés seront comparés à ceux proposés par Pettit et

Revue internationale P.M.E., vol. 14, n 1, 2001 
Singer (1985) afin de déterminer dans quelle mesure leurs recommandations ont porté des fruits. Nous relèverons finalement les limites de nos résultats et proposerons des voies de recherche.

\section{Cadre opératoire}

Nous décrirons ici les aspects méthodologiques de notre étude. En premier lieu, nous présenterons les revues scientifiques sélectionnées. Nous décrirons ensuite le mode de sélection des articles étudiés et exposerons les méthodes d'identification et de catégorisation des objets de recherche répertoriés.

Les articles étudiés proviennent de six revues scientifiques de renom dans le domaine de l'entrepreneuriat et de la PME : Entrepreneurship Theory and Practice (ETP), International Small Business Journal (ISBJ), Journal of Business Venturing (JBV), Journal of Small Business Management (JSBM), Revue internationale PME (RIPME) et Small Business Economics (SBE). Les cinq revues anglophones ont fait l'objet d'une évaluation par des chercheurs chevronnés dans le domaine de l'entrepreneurship et de la gestion des PME et ont été classées parmi les 24 revues les plus appropriées comme tribune pour la publication de travaux de recherche dans ce champ de connaissances (MacMillan, 1993). La revue JBV est la plus prestigieuse sur l'échelle de MacMillan avec une cote de 3,42 sur 4, alors que les revues ETP, SBE, JSBM et ISBJ obtiennent des évaluations de 2,61, 2,33, 2,05 et 1,89 respectivement. La RIPME a été choisie parce qu'elle est la seule revue scientifique francophone consacrée exclusivement à la PME. Notons que nous n'avons pas inclus les articles publiés dans la revue ISBJ avant 1992, lorsque celle-ci portait le nom de European Small Business Journal (ESBJ), d'autant plus que les textes de ESBJ ne sont pas répertoriés dans la base de données que nous avons utilisée.

Pour toutes les revues, à l'exception de RIPME et de SBE pour les années 1989 à 1991, la sélection des articles a été effectuée à partir de la banque de données ABI / INFORM. Cette base de données informatisée répertorie près de 1000 revues anglophones spécialisées provenant des quatre coins du monde. L'interface de recherche permet d'interroger la banque de données selon certains paramètres précis. Les paramètres de sélection qui ont été utilisés sont les suivants : le titre de la publication, l'année de publication de l'article et le sujet de l'article. Les revues visées sont celles mentionnées précédemment. La recherche a débuté en janvier 1989 pour se terminer en septembre 1998. Le paramètre sujet a été défini en utilisant le terme «financ ? (le point d'interrogation remplace toute autre lettre ou groupe de lettres; ainsi les mots «finance » et «financier» seront considérés). Ce terme a permis de faire ressortir tous les articles traitant de finance dans les revues ciblées. La RIPME et la revue SBE pour les années 1989 à 1991 ne sont pas répertoriés par ABI/INFORM. Dans le cas de RIPME, pour la période de 1989 à 1995, la sélection des articles est basée sur la catégorisation par discipline des travaux publiés 
dans cette revue, effectuée par Pierre Cossette (1996). La sélection des articles pour les autres années dans RIPME et pour SBE découle du jugement de l'auteur du présent document.

Au total, 125 articles répondaient aux critères stipulés. Tous les objets de recherche ont été identifiés à partir du texte intégral des articles recueillis. Tous les articles ont été imprimés et conservés pour en permettre l'analyse ultérieure. Dix-sept articles ont été considérés comme ne faisant pas état d'une recherche dite scientifique; ils ont été éliminés avant de procéder à l'analyse des données. Ces articles constituaient des études de cas, des critiques de monographies et des textes écrits sous les vocables «Executive Forum » et «Small Business News \& Views ».

Les objets de recherche retenus ont été regroupés en 11 catégories : le capital de risque (CAPR), la création d'entreprises (CENT), les conditions de financement (CFIN), l'entrepreneurship (ENTR), l'évaluation (EVAL), la gestion financière (GFIN), l'objectif financier (OBJE), la performance financière (PERF), la recherche $(\mathrm{RECH})$, la structure de capital (SCAP) et la structure organisationnelle (SORG). Ces catégories ont été déterminées par l'auteur du présent article et correspondent à des thèmes fréquemment rencontrés en finance moderne. L'annexe I présente la liste de tous les objets de recherche classés par catégorie, par revue et par année.

\section{Résultats}

Les résultats de cette étude portent sur les articles répertoriés faisant état d'une recherche scientifique dans l'une ou l'autre des six revues scientifiques mentionnées précédemment. Quatre tableaux permettront de dégager les caractéristiques des objets de recherche répertoriés. Le tableau 1 présente le nombre d'articles publiés par revue et leur évolution au cours des 10 dernières années.

\section{TABLEAU 1}

\section{Nombre d'articles publiés par revue et évolution au cours des $\mathbf{1 0}$ dernières années}

\begin{tabular}{lcccccccccccc}
\hline & $\mathbf{1 9 8 9}$ & $\mathbf{1 9 9 0}$ & $\mathbf{1 9 9 1}$ & $\mathbf{1 9 9 2}$ & $\mathbf{1 9 9 3}$ & $\mathbf{1 9 9 4}$ & $\mathbf{1 9 9 5}$ & $\mathbf{1 9 9 6}$ & $\mathbf{1 9 9 7}$ & $\mathbf{1 9 9 8}$ & Total & $\%$ \\
\hline ETP & & 1 & & 2 & 1 & & 3 & & 1 & & 8 & 7,4 \\
ISBJ & & & & 2 & 1 & 1 & 1 & 2 & & & 7 & 6,5 \\
JBV & 1 & 1 & 1 & 3 & 1 & 1 & 1 & 1 & & 2 & 12 & 11,1 \\
JSBM & 3 & & 4 & 4 & 5 & 3 & 2 & 1 & 3 & 2 & 27 & 25,0 \\
RIPME & 1 & 1 & 2 & 4 & 1 & & 10 & 3 & 1 & 1 & 24 & 22,2 \\
SBE & 3 & 5 & 4 & 1 & 3 & 2 & 5 & & 5 & 2 & 30 & 27,8 \\
\hline Total & 8 & 8 & 11 & 16 & 12 & 7 & 22 & 7 & 10 & 7 & 108 & 100,0 \\
\hline \%) & 7,4 & 7,4 & 10,2 & 14,8 & 11,1 & 6,4 & 20,4 & 6,5 & 9,3 & 6,5 & 100,0 \\
\hline
\end{tabular}


D'entrée de jeu, on constate que les revues JSBM, RIPME et SBE regroupent 81 articles représentant $75 \%$ du total de tous les articles répertoriés; ces revues publient des articles sur la finance des PME de façon régulière durant la période observée, avec une pointe en 1995 due à la publication d'un numéro spécial de la revue RIPME portant sur le financement des PME. Les trois autres revues (ETP, ISBJ et JBV) sont beaucoup moins prolifiques; il faut toutefois noter que la revue ISBJ n'est répertoriée que depuis 1992.

Le tableau 2 présente le nombre d'auteurs qui ont été répertoriés durant la période observée en relation avec le nombre d'articles qu'ils ont publiés.

TABLEAU 2

Nombre d'articles par auteur

\begin{tabular}{ccc}
\hline Nombre d'articles & Nombre d'auteurs & Pourcentage \\
\hline 1 & 124 & 79,5 \\
2 & 28 & 17,9 \\
3 & 1 & 0,7 \\
4 & 3 & 1,9 \\
\hline Total & 156 & 100,0 \\
\hline
\end{tabular}

Nous constatons qu'il existe une grande diversité d'auteurs dans le domaine de la finance des PME. La majorité des auteurs ne semblent pas porter un intérêt soutenu pour la recherche en finance des PME : 79,5\% des auteurs n'ont publié qu'un seul article, $17,9 \%$ ont publié deux articles et seulement $2,6 \%$ ont publié trois ou quatre articles.

Le tableau 3 présente le nombre d'objets de recherche par catégorie et par année. Les objets de recherche les plus fréquents au cours de la période étudiée sont les conditions de financement (CFIN) et la performance financière (PERF). Trente-neuf articles $(36,1 \%)$ ont porté sur les conditions de financement alors que trente études $(27,7 \%)$ ont traité de la performance des PME. Sous le premier thème, les recherches portent principalement sur l'offre de financement, l'accessibilité au financement et la description des modes de financement utilisés par les PME. Les études sur la performance financière traitent principalement de l'impact de différents facteurs sur la performance des PME et sur l'évaluation de la performance financière.

La gestion financière (GFIN) vient en troisième lieu avec 12 articles pour $11,1 \%$ du total. Dans cette catégorie, les chercheurs s'intéressent à l'analyse des données financières des PME et aux outils, pratiques et processus financiers. Le capital de risque (CAPR) et la structure de capital (SCAP) regroupent huit articles chacun représentant 7,4\% du total. Sous le thème du capital de risque, on s'attarde 
surtout au rôle et aux facteurs qui influent sur l'offre de capital de risque alors que les études sur la structure de capital portent essentiellement sur les politiques en vigueur dans les PME et les facteurs qui les influencent.

Les autres objets de recherche que nous avons répertoriés ne comptent que très peu d'articles par catégorie. Ainsi, nous n'avons relevé que trois articles traitant de la création d'entreprises (CENT); l'évaluation (EVAL), l'objectif financier (OBJE) et la recherche (RECH) ne comptent que deux articles chacun; les objets de recherche entrepreneurship (ENTR) et structure organisationnelle (SORG) n'ont fait l'objet que d'un seul article chacun.

TABLEAU 3

Évolution des objets de recherche au cours des 10 dernières années

\begin{tabular}{|c|c|c|c|c|c|c|c|c|c|c|c|c|}
\hline & 1989 & 1990 & 1991 & 1992 & 1993 & 1994 & 1995 & 1996 & 1997 & 1998 & Total & $\%$ \\
\hline CAPR & 1 & 1 & 1 & 2 & 1 & & 2 & & & & 8 & 7,4 \\
\hline CENT & & & & 1 & & & 1 & & 1 & & 3 & 2,8 \\
\hline CFIN & 2 & 4 & 3 & 7 & & 3 & 11 & 1 & 5 & 3 & 39 & 36,1 \\
\hline ENTR & & & & & 1 & & & & & & 1 & 0,9 \\
\hline EVAL & & & 1 & & 1 & & & & & & 2 & 1,9 \\
\hline GFIN & 1 & & 2 & 3 & 2 & 1 & 1 & 1 & 1 & & 12 & 11,1 \\
\hline OBJE & & & & & & & 1 & 1 & & & 2 & 1,9 \\
\hline PERF & 3 & 2 & 3 & 2 & 4 & 3 & 3 & 3 & 3 & 4 & 30 & 27,7 \\
\hline $\mathrm{RECH}$ & & & & 1 & & & & 1 & & & 2 & 1,9 \\
\hline SCAP & 1 & 1 & 1 & & 2 & & 3 & & & & 8 & 7,4 \\
\hline SORG & & & & & 1 & & & & & & 1 & 0,9 \\
\hline Total & 8 & 8 & 11 & 16 & 12 & 7 & 22 & 7 & 10 & 7 & 108 & 100,0 \\
\hline$(\%)$ & 7,4 & 7,4 & 10,2 & 14,8 & 11,1 & 6,5 & 20,4 & 6,5 & 9,2 & 6,5 & 5100,0 & \\
\hline
\end{tabular}

Nous concluons que deux domaines particuliers retiennent l'attention des chercheurs de façon soutenue : les conditions de financement (CFIN) et la performance financière (PERF); ces objets de recherche représentent $63,8 \%$ des objets de recherche que nous avons recensés. Parmi les autres objets de recherche, notons que la gestion financière (GFIN), le capital de risque (CAPR) et la structure de capital (SCAP) ont engendré $25,9 \%$ des études alors que les autres objets de recherche n'ont été traités que de façon sporadique. Dans la prochaine section, nous tenterons d'évaluer la pertinence de ces recherches par rapport aux problématiques existantes en matière de théorie financière pour les PME.

\section{Recommandations passées : Pettit et Singer ont-ils été entendus?}

Dans cette section, nous dégagerons des voies de recherche en finance des PME en comparant les objets de recherche que nous avons relevés au cours des 10 dernières années à ceux qui avaient été proposés à la communauté scientifique quelques 
années auparavant. Pour ce faire, nous avons relevé trois articles proposant des pistes de recherche au cours des quatre années précédant notre étude : Pettit et Singer (1985), Wortman (1987) et Low et MacMillan (1988). L'article de Pettit et Singer nous est apparu le plus pertinent comme base de comparaison pour notre recherche parce qu'il traite spécifiquement de finance des PME.

Pettit et Singer ont proposé des voies de recherche dans plusieurs domaines de la finance des PME dans un article intitulé «Small Business Finance : A Research Agenda» publié dans la revue Financial Management en 1985. Les auteurs suggèrent des domaines spécifiques de recherche pour lesquelles les contributions sont susceptibles d'augmenter significativement notre compréhension de la finance des petites entreprises. Leur objectif était de faire en sorte que les particularités des PME puissent être éventuellement intégrées dans le corpus de connaissances en finance moderne.

Les objets de recherche recommandés par Pettit et Singer étaient regroupés en deux sections : la structure de l'actif et la structure de financement. Les auteurs avaient subdivisé chaque section en catégories comme suit: le rôle de l'entrepreneur, la décision d'investissement, le coût et la disponibilité du crédit, l'accès au financement par actions, la structure de capital optimale, la politique de dividende et la forme de l'organisation. En revanche, les objets de recherche proposés apparaissaient dans le texte de façon non structurée. Dans cette étude, les sections et catégories ont été conservées, mais les questions de recherches, au nombre de 20, découlent de l'interprétation de l'auteur du présent article. Pour chaque catégorie, nous identifierons les objets de recherche qui ont été traités durant les 10 dernières années et nous ferons ressortir ceux qui ont été laissés en plan.

L'annexe II présente les voies de recherche proposées par Pettit et Singer ainsi que le nombre d'articles répertoriés dans cette étude qui traitent des objets de recherche recommandés. Toutes les recommandations ont été numérotées. Les articles répertoriés peuvent être retracés à l'annexe I en repérant le numéro de la recommandation dans la deuxième colonne $(\mathrm{P} \& \mathrm{~S})$. Le tableau 4 présente un résumé de l'annexe II et identifie le nombre d'articles qui traitent des recommandations de Pettit et Singer de façon spécifique ou générale.

\subsection{La structure de l'actif}

\subsubsection{Le rôle de l'entrepreneur}

Quatre articles portent sur l'impact de l'entrepreneur sur la performance financière des PME. Les textes relevés ne permettent cependant pas de déterminer, comme l'avaient proposé Pettit et Singer, si l'actif entrepreneurial est plus important dans les PME, ni de connaître la valeur et la composition du portefeuille des propriétairesdirigeants en rapport avec les choix d'investissement de la firme. 


\section{TABLEAU 4}

Comparaison des articles répertoriés aux objets de recherche proposés par Pettit et Singer

\begin{tabular}{llccc}
\hline & Objets proposés & \multicolumn{3}{c}{ Articles répertoriés } \\
\hline & & $\begin{array}{c}\text { Portée } \\
\text { spécifique }\end{array}$ & $\begin{array}{c}\text { Portée } \\
\text { générale }\end{array}$ & $\begin{array}{c}\text { Autres } \\
\text { objets }\end{array}$ \\
\hline 1 & LA STRUCTURE DE L'ACTIF & & & \\
1.1 & Le rôle de l'entrepreneur & & \\
1.2 & La décision d'investissement & & & \\
\hline 2 & LA STRUCTURE DE FINANCEMENT & & 27 & \\
2.1 & Le coût et la disponibilité du crédit & 8 & 2 & \\
2.2 & L'accès au financement par actions & 1 & 5 & \\
2.3 & La structure de capital optimale & 6 & 1 & \\
2.4 & La politique de dividende & & & \\
2.5 & La forme de l'organisation & & 1 & 49,1 \\
\hline & Total & 15 & 40 & \\
\hline & (\%) & 13,9 & 37,0 & \\
\hline
\end{tabular}

\subsubsection{La décision d'investissement}

Aucun article n'a porté sur la décision d'investissement. Toutes les questions amenées par Pettit et Singer restent à être élucidées : si l'information asymétrique et la flexibilité sont plus grandes dans la PME, engendrant un problème d'estimation plus grand pour les intervenants externes, alors comment le problème est-il résolu et comment la résolution de ce problème dans les PME diffère-t-elle de celle des plus grandes entreprises? Y a-t-il des restrictions contractuelles qui tendent à limiter la flexibilité des PME ? Est-ce que l'information asymétrique amène à prendre des décisions d'investissement sous-optimales? Quelles sont les méthodes utilisées par les intervenants externes pour évaluer le risque des PME ? Est-ce que les PME ont tendance à avoir un niveau de risque plus élevé que les plus grandes entreprises et est-ce que le problème d'estimation de ce risque est plus grand?

\subsection{La structure de financement}

Nous avons relevé 27 articles traitant de la structure de financement des PME; cependant, l'optique de ces recherches ne nous a pas permis de les relier à l'une ou l'autre des questions spécifiques de cette section. 


\subsubsection{Le coût et la disponibilité du crédit}

Deux articles portent sur le coût de financement pour les PME, mais ne permettent pas de répondre aux questions soulevées par Pettit et Singer. Les articles de Binks, Ennew et Reed (1992), Psillaki (1995) et Cowling et Clay (1995) traitent de l'impact de l'information asymétrique sur le coût de la dette et de la disponibilité des fonds sous forme d'emprunt pour la PME. Les articles des auteurs suivants portent sur la discrimination possible dont souffrent les PME de la part des institutions financières en ce qui a trait au coût de la dette et à la disponibilité des fonds : Levratto (1990), Diouf et Wade (1992), Jullien et Paranque (1995), Audretsch et Elston (1995) et Cieply et Hancké (1998). Les questions suivantes restent en plan : sachant que les PME utilisent davantage le mode de financement par dette, quels sont les déterminants de cette orientation? Est-ce que les coûts d'agence sont plus importants dans la PME que dans la plus grande entreprise? Quelles sont les caractéristiques des contrats d'emprunt des PME?

\subsubsection{L'accès au financement par actions}

Cinq articles traitent de l'accès au financement par actions mais ne permettent pas de répondre aux autres questions soulevées par Pettit et Singer dans cette catégorie. L'article de Hustedde et Pulver (1992) porte sur les caractéristiques des PME qui obtiennent du financement par actions sur les marchés privés, incluant les firmes de capital de risque. Les questions suivantes demeurent sans réponses : existe-t-il un lien entre le niveau d'asymétrie de l'information et le niveau de financement par actions utilisé par les PME? Quelles sont les caractéristiques de propriété et de contrôle des PME? Quels sont les problèmes d'agence des PME qui ont des propriétaires multiples et de quelle façon ces problèmes sont-ils efficacement réglés?

\subsubsection{La structure de capital optimale}

Un article porte sur la structure de capital mais ne permet pas de répondre aux questions soulevées par Pettit et Singer. Nous avons répertorié six articles traitant du niveau optimal d'emprunt pour les PME. Il s'agit des textes de Barton et Matthews (1989), Norton (1991), Merikas, Bruton et Vozikis (1993), Van der Wijst et Thurik (1993), Chaganti, DeCarolis et Deeds (1995) et Cressy (1995). Ces auteurs relèvent différents facteurs qui influencent les décisions en matière de structure de capital dans les PME.

\subsubsection{La politique de dividende et la forme de l'organisation}

Un article porte sur la forme de l'organisation mais ne permet pas de répondre aux questions soulevées par Pettit et Singer. Nous n'avons répertorié aucun article sur la politique de dividende ou sur la forme de l'organisation. Les questions suivantes

Revue internationale P.M.E., vol. 14, nº 1, 2001 
restent en suspens : quelles sont les causes exactes du niveau de dividendes moins élevé dans les PME ? Est-ce que la théorie d'effet d'annonce relative aux dividendes est applicable aux PME? Quel est l'impact de la forme d'organisation adoptée sur les coûts de financement de la PME?

En résumé, seulement 15 articles $(13,9 \%)$ portent sur des questions spécifiques alors que 40 articles $(37 \%)$ traitent de façon générale des objets proposés par Pettit et Singer. Les 53 articles restants $(49,1 \%)$ sont basés sur des objets de recherche autres que ceux qui ont été proposés. Seulement 4 des 20 questions soulevées par Pettit et Singer ont été abordées au cours de la période visée par notre étude. On constate par ailleurs, en interrogeant la base de données, que Pettit et Singer n'ont publié jusqu'à ce jour aucun article traitant des objets de recherche qu'ils avaient recommandés.

\section{Conclusion}

Notre étude a permis de constater qu'il existe une grande diversité d'auteurs dans le domaine de la recherche sur la finance des PME et qu'ils n'y portent pas un intérêt soutenu. Les revues JSBM, RIPME et SBE publient la majorité des articles en finance des PME, et ce, de façon très assidue alors que les revues ETP et ISBJ et JBV publient relativement moins d'articles.

Les recherches effectuées portent principalement sur deux objets de recherche : les conditions de financement et la performance financière. Les thèmes les plus fréquemment abordés sont l'offre de financement, l'accessibilité au financement, la description des modes de financement utilisés, l'impact de différents facteurs sur la performance et l'évaluation de la performance financière des PME. Les chercheurs s'intéressent aussi, mais dans une moindre mesure, à l'analyse des données financières, aux outils, pratiques et processus financiers et aux facteurs qui influencent l'offre de capital de risque et la structure de capital des PME.

Les voies de recherche en finance des PME proposées par Pettit et Singer en 1985 ne semblent pas avoir suscité l'intérêt des chercheurs dans ce champ de connaissances. Les impacts de l'asymétrie de l'information, de la flexibilité des opérations et des coûts d'agence sur la décision d'investissement, le risque perçu et la structure de financement des PME demeurent des objets de recherche pratiquement ignorés par la communauté scientifique. Aucun développement ne s'est effectué au cours des 10 dernières années au regard des particularités associées à la politique de dividende des PME et à l'impact de la forme de l'organisation sur les coûts de financement des PME.

Comme voie additionnelle de recherche, il nous semblerait intéressant de tenter de découvrir les raisons d'un tel désintéressement de la part de la communauté scientifique pour le domaine de la finance des PME et, plus particulièrement, 
en ce qui a trait aux questions de recherche soulevées par Pettit et Singer en 1985. Existe-t-il des difficultés importantes d'accès aux données ou de méthodologie qui empêchent les chercheurs de mener à bien de telles études? Les résultats obtenus jusqu'à maintenant sont-ils assez intéressants et suffisamment spécifiques à la PME pour justifier des études additionnelles ? Existe-t-il suffisamment de sources de fonds pour mener à bien de telles recherches?

La présente étude comporte certaines limites. La première concerne la méthode de classification utilisée par ABI / INFORM pour codifier les sujets des articles répertoriés; nous n'avons pas validé cette codification et il est donc possible que certains articles traitant de finance aient été codés sous un autre vocable. La deuxième limite concerne le choix d'un nombre restreint de revues scientifiques ; l'utilisation d'un plus grand nombre de revues aurait pu donner des résultats différents. La dernière limite a trait à l'interprétation qu'a fait l'auteur des objets de recherche répertoriés ; une interprétation différente des objets de recherche par un autre chercheur aurait pu produire des résultats différents. Néanmoins, nous croyons que cette étude a permis de dresser un premier bilan de la recherche en finance des PME et de souligner à nouveau l'importance de poursuivre les recherches dans ce domaine

ANNEXE I

Caractéristiques des articles sélectionnés

\begin{tabular}{|c|c|c|c|c|}
\hline Fran* P \& S & Revue & Année & Auteur & Objets \\
\hline CAPR 2.2 & SBE & 1989 & Gaston & $\begin{array}{l}\text { L'importance du marché de capital de risque } \\
\text { informel aux États-Unis }\end{array}$ \\
\hline CAPR & SBE & 1990 & Yazdipour & $\begin{array}{l}\text { La performance des entreprises de capital de } \\
\text { risque aux États-Unis }\end{array}$ \\
\hline CAPR & SBE & 1991 & Flynn & $\begin{array}{l}\text { Les facteurs affectant les relations entre les } \\
\text { entreprises de capital de risque } \\
\text { et les entrepreneurs }\end{array}$ \\
\hline CAPR & JBV & 1992 & Gupta et Sapienza & $\begin{array}{l}\text { Les facteurs affectant les décisions } \\
\text { d'investissement des firmes de capital } \\
\text { de risque }\end{array}$ \\
\hline CAPR & JSBM & 1992 & Norton et Tenenbaum & $\begin{array}{l}\text { Les facteurs affectant le type de financement } \\
\text { offert par les entreprises de capital de risque }\end{array}$ \\
\hline CAPR & JSBM & 1993 & Norton et Tenenbaum & $\begin{array}{l}\text { L'impact des caractéristiques des entreprises } \\
\text { de capital de risque sur le type } \\
\text { de financement qu'elles offrent }\end{array}$ \\
\hline CAPR 2.2 & RIPME & 1995 & Suret et Arnoux & $\begin{array}{l}\text { Le capital de risque direct : origines, } \\
\text { formes et importance }\end{array}$ \\
\hline CAPR & SBE & 1995 & Mason et Harrison & $\begin{array}{l}\text { Le rôle des pourvoyeurs informels } \\
\text { de capital de risque }\end{array}$ \\
\hline CENT & SBE & 1992 & Felsenstein & $\begin{array}{l}\text { L'impact de programmes de financement } \\
\text { offerts aux PME sur la création d'emploi } \\
\text { en Israël }\end{array}$ \\
\hline CENT & JBV & 1995 & Gunther et McGrath & $\begin{array}{l}\text { La façon dont les entreprises réagissent } \\
\text { aux échecs des nouvelles entreprises } \\
\text { qu'elles créent }\end{array}$ \\
\hline
\end{tabular}

Revue internationale P.M.E., vol. 14, $\mathrm{n}^{\circ}$ 1, 2001 
ANNEXe I (suite)

Caractéristiques des articles sélectionnés

\begin{tabular}{|c|c|c|c|c|c|}
\hline Fran* & $\mathbf{P} \& \mathbf{S}$ & Revue & Année & Auteur & Objets \\
\hline CENT & & SBE & 1997 & Egeln et al. & $\begin{array}{l}\text { Les déterminants de la création d'entreprises } \\
\text { en Allemagne de l'Ouest }\end{array}$ \\
\hline CFIN & & RIPME & 1989 & Desroches et Jog & $\begin{array}{l}\text { Les effets du financement public } \\
\text { sur les PME au Québec }\end{array}$ \\
\hline CFIN & 2 & SBE & 1989 & Walker & $\begin{array}{l}\text { Les caractéristiques des besoins } \\
\text { en financement des PME }\end{array}$ \\
\hline CFIN & 2.2 & JBV & 1990 & Freear et Wetzel & $\begin{array}{l}\text { L'identification des sources de financement } \\
\text { en actions des entreprises de technologie } \\
\text { de pointe en émergence }\end{array}$ \\
\hline CFIN & 2.1 .2 & RIPME & 1990 & Levratto & $\begin{array}{l}\text { Le financement des PME par les banques } \\
\text { en France }\end{array}$ \\
\hline CFIN & 2 & SBE & 1990 & Rapoport & $\begin{array}{l}\text { Le financement des innovations } \\
\text { technologiques dans les PME }\end{array}$ \\
\hline CFIN & 2 & SBE & 1990 & Rappaport et Wyatt & $\begin{array}{l}\text { La participation des banques aux } \\
\text { programmes de prêts aux PME garantis } \\
\text { par le gouvernement }\end{array}$ \\
\hline CFIN & 2 & JSBM & 1991 & Chotigeat et al. & $\begin{array}{l}\text { Les sources de financement des entrepreneurs } \\
\text { asiatiques établis aux États-Unis }\end{array}$ \\
\hline CFIN & & RIPME & 1991 & Belletante & Les spécificités financières des PME \\
\hline CFIN & & RIPME & 1991 & Desroches et al. & $\begin{array}{l}\text { Les effets du financement public } \\
\text { sur les PME au Québec }\end{array}$ \\
\hline CFIN & 2.1 .1 & ISBJ & 1992 & Binks et al. & $\begin{array}{l}\text { L'accès au financement par les PME } \\
\text { et l'information asymétrique }\end{array}$ \\
\hline CFIN & 2.2 .2 & JBV & 1992 & Hustedde et Pulver & $\begin{array}{l}\text { Les facteurs affectant l'obtention } \\
\text { de financement par actions }\end{array}$ \\
\hline CFIN & 2 & JSBM & 1992 & Beedles & $\begin{array}{l}\text { Le coût du financement par actions } \\
\text { des PME en Australie }\end{array}$ \\
\hline CFIN & 2 & RIPME & 1992 & Fauré & Le financement des PME en Côte-d'Ivoire \\
\hline CFIN & 2 & RIPME & 1992 & Gnansounou & Les tontines et l'artisanat au Bénin \\
\hline CFIN & 2.1 & RIPME & 1992 & Haddab et Traimond & $\begin{array}{l}\text { Les institutions financières islamiques } \\
\text { et les besoins en fonds de roulement des PME }\end{array}$ \\
\hline CFIN & 2.1 .2 & RIPME & 1992 & Diouf et Wade & $\begin{array}{l}\text { Le financement des PME par les banques } \\
\text { au Sénégal }\end{array}$ \\
\hline CFIN & 2.2 & ISBJ & 1994 & Murray & $\begin{array}{l}\text { Les problèmes reliés à l'obtention } \\
\text { de financement par actions des entreprises } \\
\text { en démarrage }\end{array}$ \\
\hline CFIN & 2.1 & JSBM & 1994 & Holmes et al. & Le coût de la dette pour les PME en Australie \\
\hline CFIN & 2 & SBE & 1994 & Keasey et Watson & $\begin{array}{l}\text { La disponibilité et les caractéristiques du } \\
\text { financement bancaire en Grande-Bretagne }\end{array}$ \\
\hline CFIN & 2 & ISBJ & 1995 & Deakins et Philpott & $\begin{array}{l}\text { L'offre de financement aux PME par les } \\
\text { réseaux d'institutions financières } \\
\text { et d'agence de support en Europe }\end{array}$ \\
\hline CFIN & 2 & RIPME & 1995 & Picory & $\begin{array}{l}\text { L'évaluation du risque des PME } \\
\text { par les banques }\end{array}$ \\
\hline CFIN & 2 & RIPME & 1995 & Allegret & $\begin{array}{l}\text { Les technopoles comme réponse } \\
\text { aux contraintes de financement } \\
\text { des PME innovantes }\end{array}$ \\
\hline CFIN & 2 & RIPME & 1995 & Marion & $\begin{array}{l}\text { Le financement de l'actif } \\
\text { immatériel des nouvelles entreprises } \\
\text { technologiques et innovantes }\end{array}$ \\
\hline
\end{tabular}

Revue internationale P.M.E., vol. 14, n 1, 2001 


\section{AnNEXe I (suite) \\ Caractéristiques des articles sélectionnés}

\begin{tabular}{|c|c|c|c|c|c|}
\hline Fran* & $P \& S$ & Revue & Année & Auteur & Objets \\
\hline CFIN & 2.1 .1 & RIPME & 1995 & Psillaki & $\begin{array}{l}\text { Rationnement du crédit aux PME } \\
\text { et information asymétrique }\end{array}$ \\
\hline CFIN & 2.1 .2 & RIPME & 1995 & Jullien et Paranque & $\begin{array}{l}\text { Le financement des PME et l'évolution } \\
\text { du système financier }\end{array}$ \\
\hline CFIN & 2.1 .2 & RIPME & 1995 & Audretsch et Elston & $\begin{array}{l}\text { Le système de financement aux PME } \\
\text { en Allemagne }\end{array}$ \\
\hline CFIN & 2.1 .1 & SBE & 1995 & Cowling et Clay & $\begin{array}{l}\text { Les facteurs qui influencent l'obtention } \\
\text { de prêts bancaires garantis par le } \\
\text { gouvernement en Grande-Bretagne }\end{array}$ \\
\hline CFIN & 2.2 & SBE & 1995 & Keasey et McGuiness & $\begin{array}{l}\text { Une revue des concepts reliés à la } \\
\text { sous-évaluation des prix des actions } \\
\text { lors de premières émissions publiques }\end{array}$ \\
\hline CFIN & & RIPME & 1996 & Belletante et Desroches & $\begin{array}{l}\text { Les effets de l'accès au financement public } \\
\text { par des PME sur leur comportement financier }\end{array}$ \\
\hline CFIN & 2 & ETP & 1997 & Binks et Ennew & $\begin{array}{l}\text { Les interrelations entre les PME } \\
\text { et les banques }\end{array}$ \\
\hline CFIN & 2 & SBE & 1997 & Cressy et Olofsson & Les conditions financières des PME en Suède \\
\hline CFIN & 2 & SBE & 1997 & Manigart et Struyf & $\begin{array}{l}\text { Le financement des entreprises de haute } \\
\text { technologie en démarrage en Belgique }\end{array}$ \\
\hline CFIN & 2 & SBE & 1997 & Audretsh et Elston & $\begin{array}{l}\text { Les conditions de financement } \\
\text { des PME en Allemagne }\end{array}$ \\
\hline CFIN & 2 & SBE & 1997 & Hughes & $\begin{array}{l}\text { Les conditions de financement } \\
\text { des PME en Grande-Bretagne }\end{array}$ \\
\hline CFIN & 2.1 .2 & RIPME & 1998 & Cieply et Hancké & $\begin{array}{l}\text { L'hypothèse de rationnement } \\
\text { de crédit aux PME de la part des banques }\end{array}$ \\
\hline CFIN & 2 & SBE & 1998 & Deeg & $\begin{array}{l}\text { Les caractéristiques distinctives } \\
\text { du système bancaire en Allemagne }\end{array}$ \\
\hline CFIN & 2 & SBE & 1998 & Vitols & $\begin{array}{l}\text { Les caractéristiques distinctives } \\
\text { du système bancaire en Allemagne }\end{array}$ \\
\hline ENTR & & RIPME & 1993 & $\mathrm{Yu}$ & $\begin{array}{l}\text { Une analyse de la décision d'investissement } \\
\text { d'entrepreneurs chinois fonctionnant } \\
\text { dans un système de responsabilité }\end{array}$ \\
\hline EVAL & & JSBM & 1991 & Burns et Walker & $\begin{array}{l}\text { L'utilisation de la méthode d'actualisation } \\
\text { des flux monétaires lorsque l'évaluation } \\
\text { dépend de l'amortissement }\end{array}$ \\
\hline EVAL & & JSBM & 1993 & Baucus et al. & $\begin{array}{l}\text { La relation entre le coût initial, } \\
\text { les redevances et la valeur d'une franchise }\end{array}$ \\
\hline GFIN & & JSBM & 1989 & Chen (K.C.) & $\begin{array}{l}\text { Le développement de systèmes d'aide } \\
\text { à la décision pour les gestionnaires de PME }\end{array}$ \\
\hline GFIN & & JSBM & 1991 & McMahon et Holmes & $\begin{array}{l}\text { Les pratiques en matière de gestion financière } \\
\text { en Amérique du Nord }\end{array}$ \\
\hline GFIN & & JSBM & 1991 & Weinrauch et al. & $\begin{array}{l}\text { Les perceptions et attitudes des gestionnaires } \\
\text { de PME concernant les budgets limités } \\
\text { de marketing }\end{array}$ \\
\hline GFIN & & ISBJ & 1992 & Laitinen & $\begin{array}{l}\text { Les processus financiers dans les PME } \\
\text { et le risque de faillite }\end{array}$ \\
\hline GFIN & & JSBM & 1992 & Coker et Hayes & $\begin{array}{l}\text { La perception des créanciers face } \\
\text { aux états financiers préparés sur la base } \\
\text { des revenus imposables }\end{array}$ \\
\hline GFIN & & JSBM & 1992 & Osteryoung et al. & $\begin{array}{l}\text { Une comparaison de ratios financiers } \\
\text { de petites et grandes entreprises }\end{array}$ \\
\hline
\end{tabular}

Revue internationale P.M.E., vol. 14, $\mathrm{n}^{\circ}$ 1, 2001 
ANNEXe I (suite)

\section{Caractéristiques des articles sélectionnés}

\begin{tabular}{|c|c|c|c|c|c|}
\hline Fran* & $\mathbf{P} \& \mathbf{S}$ & Revue & Année & Auteur & Objets \\
\hline GFIN & & ETP & 1993 & Brophy et Shulman & $\begin{array}{l}\text { Un modèle de facteurs financiers } \\
\text { qui stimule l'innovation }\end{array}$ \\
\hline GFIN & 2 & JSBM & 1993 & Van Auken et al. & $\begin{array}{l}\text { L'étude des bilans de PME coréennes } \\
\text { et américaines }\end{array}$ \\
\hline GFIN & & SBE & 1994 & Keasey et Watson & $\begin{array}{l}\text { L'impact de la loi de } 1986 \text { relative à } \\
\text { l'insolvabilité et à la suspension des } \\
\text { administrateurs sur les décisions financières } \\
\text { des propriétaires-dirigeants } \\
\text { en Grande-Bretagne }\end{array}$ \\
\hline GFIN & 2 & ETP & 1995 & Auken et Holman & $\begin{array}{l}\text { L'étude de bilans } \\
\text { de petites entreprises publiques }\end{array}$ \\
\hline GFIN & & ISBJ & 1996 & Peel et Wilson & La gestion financière dans les PME \\
\hline GFIN & & JSBM & 1997 & Gray et al. & $\begin{array}{l}\text { Les caractéristiques de PME manufacturières } \\
\text { au Kenya }\end{array}$ \\
\hline OBJE & & ETP & 1995 & McMahon et Stanger & L'objectif financier des PME \\
\hline OBJE & & JSBM & 1996 & LeCornu et al. & L'objectif financier des PME \\
\hline PERF & 2 & JBV & 1989 & Bull & $\begin{array}{l}\text { La performance financière d'entreprises } \\
\text { ayant subi un LBO }\end{array}$ \\
\hline PERF & & JSBM & 1989 & Shrader et al. & $\begin{array}{l}\text { La relation entre la planification stratégique } \\
\text { et opérationnelle, l'incertitude } \\
\text { et la performance des } \mathrm{PME}\end{array}$ \\
\hline PERF & & SBE & 1989 & Storey & Les déterminants de la performance des PME \\
\hline PERF & 2 & ETP & 1990 & Carter et Auken & $\begin{array}{l}\text { La relation entre l'apport des propriétaires } \\
\text { et la performance financière des } \mathrm{PME}\end{array}$ \\
\hline PERF & & $\mathrm{SBE}$ & 1990 & Keasey et McGuinness & $\begin{array}{l}\text { Les rendements obtenus par les PME par } \\
\text { rapport aux types de financement utilisés }\end{array}$ \\
\hline PERF & & SBE & 1991 & Aiginger et Tichy & $\begin{array}{l}\text { Une comparaison des performances } \\
\text { des PME et des entreprises qui ont fait } \\
\text { l'objet de fusions }\end{array}$ \\
\hline PERF & & SBE & 1991 & Van Dalen et Thurik & $\begin{array}{l}\text { L'évaluation de la performance } \\
\text { des PME aux Pays-Bas }\end{array}$ \\
\hline PERF & & $\mathrm{SBE}$ & 1991 & Schneider & $\begin{array}{l}\text { Efficience, rentabilité et taille } \\
\text { des entreprises en Autriche }\end{array}$ \\
\hline PERF & & ETP & 1992 & Vos & $\begin{array}{l}\text { L'utilisation de ratios issus de grandes } \\
\text { entreprises pour évaluer la performance } \\
\text { des PME }\end{array}$ \\
\hline PERF & & JBV & 1992 & Laitinen & La prédiction de faillite des jeunes entreprises \\
\hline PERF & 1.1 & JBV & 1993 & Zahra & $\begin{array}{l}\text { L'impact de l'environnement } \\
\text { et de l'entrepreneurship corporatif } \\
\text { sur la performance financière }\end{array}$ \\
\hline PERF & & JSBM & 1993 & Acar & $\begin{array}{l}\text { L'impact de facteurs internes clés sur la } \\
\text { performance financière de PME en Turquie }\end{array}$ \\
\hline PERF & & JSBM & 1993 & Chowdhury et Lang & $\begin{array}{l}\text { La relation entre les redressements de PME } \\
\text { et la crise ou le déclin qui les précédait et le } \\
\text { support financier obtenu }\end{array}$ \\
\hline PERF & 2 & SBE & 1993 & Hall et Hutchinson & $\begin{array}{l}\text { La performance financière de PME } \\
\text { qui ont obtenu du financement par actions }\end{array}$ \\
\hline PERF & 1.1 & JBV & 1994 & Chaganti et Schneer & $\begin{array}{l}\text { L'impact du mode d'entrée du propriétaire } \\
\text { sur la performance financière } \\
\text { et les pratiques de gestion }\end{array}$ \\
\hline
\end{tabular}

Revue internationale P.M.E., vol. 14, nº 1, 2001 
ANNEXE I (suite)

Caractéristiques des articles sélectionnés

\begin{tabular}{|c|c|c|c|c|c|}
\hline Fran* & $P \& S$ & Revue & Année & Auteur & Objets \\
\hline PERF & & JSBM & 1994 & McMahon et Davies & $\begin{array}{l}\text { L'impact de l'analyse financière } \\
\text { et de l'utilisation de rapports financiers } \\
\text { sur la performance des PME }\end{array}$ \\
\hline PERF & & JSBM & 1994 & Arendarski et al. & $\begin{array}{l}\text { Les conditions et politiques nécessaires } \\
\text { pour assurer la croissance continue } \\
\text { des PME en Pologne }\end{array}$ \\
\hline PERF & & JSBM & 1995 & Olson et Bokor & $\begin{array}{l}\text { L'impact du processus et du contenu } \\
\text { stratégique sur la performance des petites } \\
\text { entreprises en forte croissance }\end{array}$ \\
\hline PERF & & JSBM & 1995 & Moini & $\begin{array}{l}\text { Les facteurs permettant aux PME } \\
\text { d'avoir du succès en exportation }\end{array}$ \\
\hline PERF & & RIPME & 1995 & El Louadi & $\begin{array}{l}\text { L'effet de la congruence informationnelle } \\
\text { sur la performance financière } \\
\text { des petites banques américaines }\end{array}$ \\
\hline PERF & & ISBJ & 1996 & Westhead et Storey & $\begin{array}{l}\text { La relation entre la formation } \\
\text { des dirigeants et la performance des PME }\end{array}$ \\
\hline PERF & & JBV & 1996 & Zahra & $\begin{array}{l}\text { L'impact des choix technologiques } \\
\text { et de l'environnement sur la } \\
\text { performance financière }\end{array}$ \\
\hline PERF & & RIPME & 1996 & St-Pierre et Beaudoin & $\begin{array}{l}\text { L'évaluation de la rentabilité financière d'un } \\
\text { projet d'implantation d'un système de gestion } \\
\text { juste-à-temps dans les PME manufacturières }\end{array}$ \\
\hline PERF & 1.1 & JSBM & 1997 & Kotey et Meredith & $\begin{array}{l}\text { L'impact des valeurs des propriétaires- } \\
\text { dirigeants et des stratégies utilisées } \\
\text { sur la performance de l'entreprise }\end{array}$ \\
\hline PERF & & JSBM & 1997 & Barringer & $\begin{array}{l}\text { L'impact des réseaux d'échanges } \\
\text { relationnels sur la performance des PME }\end{array}$ \\
\hline PERF & & RIPME & 1997 & Paranque et al. & $\begin{array}{l}\text { L'évaluation de la performance des PME } \\
\text { françaises et du risque de crédit }\end{array}$ \\
\hline PERF & 1.1 & JBV & 1998 & Chandler et Hanks & $\begin{array}{l}\text { L'impact des capitaux humains } \\
\text { et financiers sur la performance } \\
\text { d'entreprises en émergence }\end{array}$ \\
\hline PERF & & JBV & 1998 & Honig & $\begin{array}{l}\text { L'impact des capitaux humains, } \\
\text { financiers et sociaux sur la performance } \\
\text { de micro-entreprises jamaïcaines }\end{array}$ \\
\hline PERF & & JSBM & 1998 & DeDee et Vorbies & $\begin{array}{l}\text { L'impact des activités de retranchement } \\
\text { sur la performance des PME }\end{array}$ \\
\hline PERF & & JSBM & 1998 & Horng et Chen & $\begin{array}{l}\text { Les facteurs influençant l'orientation } \\
\text { de marché et l'impact de celle-ci } \\
\text { sur la performance des PME à Taiwan }\end{array}$ \\
\hline $\mathrm{RECH}$ & & ETP & 1992 & Brophy et Shulman & $\begin{array}{l}\text { La prise en compte de la théorie } \\
\text { financière dans la recherche sur la PME } \\
\text { et l'entrepreneuriat }\end{array}$ \\
\hline $\mathrm{RECH}$ & & RIPME & 1996 & Colot et Michel & $\begin{array}{l}\text { L'évolution de la recherche scientifique } \\
\text { en finance des PME }\end{array}$ \\
\hline SCAP & 2.3 .1 & JSBM & 1989 & Barton et Matthews & $\begin{array}{l}\text { L'impact de la gestion stratégique } \\
\text { sur la structure de capital des PME }\end{array}$ \\
\hline SCAP & & SBE & 1990 & Norton & $\begin{array}{l}\text { Un sondage sur les politiques en vigueur dans } \\
\text { les petites et grandes entreprises en ce qui a } \\
\text { trait à la structure de capital }\end{array}$ \\
\hline
\end{tabular}

Revue internationale P.M.E., vol. 14, nº 1, 2001 
ANNEXE I (suite)

\section{Caractéristiques des articles sélectionnés}

\begin{tabular}{|c|c|c|c|c|c|}
\hline Fran* & $P \& S$ & Revue & Année & Auteur & Objets \\
\hline SCAP & 2.3 .1 & JBV & 1991 & Norton & $\begin{array}{l}\text { Les facteurs influençant les décisions } \\
\text { relatives à la structure de capital des PME }\end{array}$ \\
\hline SCAP & 2.3 .1 & ISBJ & 1993 & Merikas et al. & $\begin{array}{l}\text { La relation entre l'objectif de la croissance } \\
\text { des ventes et la politique en matière de } \\
\text { structure de capital }\end{array}$ \\
\hline SCAP & 2.3 .1 & SBE & 1993 & Van der Wijst et Thurik & $\begin{array}{l}\text { Les déterminants de la structure } \\
\text { de capital des PME }\end{array}$ \\
\hline SCAP & 2.3 .1 & ETP & 1995 & Chaganti et al. & $\begin{array}{l}\text { L'analyse des variables qui affectent } \\
\text { les décisions relatives à la structure } \\
\text { de capital des PME }\end{array}$ \\
\hline SCAP & 2 & RIPME & 1995 & Suret et Arnoux & $\begin{array}{l}\text { Le niveau d'endettement des PME } \\
\text { québécoises }\end{array}$ \\
\hline SCAP & 2 & RIPME & 1995 & St-Pierre et Beaudoin & $\begin{array}{l}\text { Le niveau d'endettement des PME } \\
\text { québécoises }\end{array}$ \\
\hline SCAP & 2.3 & SBE & 1995 & Hutchinson & $\begin{array}{l}\text { L'impact de l'attitude des propriétaires- } \\
\text { dirigeants face au risque et la structure } \\
\text { de capital des PME }\end{array}$ \\
\hline SCAP & 2.3 .1 & SBE & 1995 & Cressy & $\begin{array}{l}\text { Un modèle d'utilisation de l'endettement } \\
\text { des PME incluant la notion de contrôle }\end{array}$ \\
\hline SORG & 2.5 & SBE & 1993 & Shailer & $\begin{array}{l}\text { La non-pertinence des frontières } \\
\text { organisationnelles des firmes appartenant } \\
\text { à des propriétaires-dirigeants }\end{array}$ \\
\hline
\end{tabular}

* Fran: Classification de l'auteur du présent article.

P \& S : Recommandations de Pettit et Singer (voir annexe II).

\section{ANNEXE II}

\section{Nombre d'articles répertoriés correspondant aux recommandations de Pettit et Singer}

\footnotetext{
Recommandations de Pettit et Singer

Nombre

d'articles

$1 \quad$ LA STRUCTURE DE L'ACTIF

1.1 Le rôle de l'entrepreneur

1.1.1 Est-ce que l'actif entrepreneurial est plus important dans la PME?

1.1.2 Quelles sont la valeur et la composition du portefeuille des propriétaires-dirigeants?

1.2 La décision d'investissement

1.2.1 Comment le problème d'estimation pour les intervenants externes face à l'information asymétrique et à la flexibilité des PME est-il résolu ?

1.2.2 Y a-t-il des restrictions contractuelles qui tendent à limiter la flexibilité des PME?

1.2.3 Est-ce que l'information asymétrique amène à prendre des décisions d'investissement sous-optimales?

1.2.4 Quelles sont les méthodes utilisées par les intervenants externes pour évaluer le risque des PME?

1.2.5 Est-ce que les PME possèdent un niveau de risque plus élevé que les plus grandes entreprises et est-ce que le problème d'estimation de ce risque est plus grand?
}

Revue internationale P.M.E., vol. 14, $\mathrm{n}^{\mathrm{o}} 1,2001$ 


\section{ANNEXE II (suite) \\ Nombre d'articles répertoriés correspondant aux recommandations de Pettit et Singer}

\begin{tabular}{|c|c|c|}
\hline & Recommandations de Pettit et Singer & $\begin{array}{l}\text { Nombre } \\
\text { d'articles }\end{array}$ \\
\hline 2 & LA STRUCTURE DE FINANCEMENT & 27 \\
\hline 2.1 & Le coût et la disponibilité du crédit & 2 \\
\hline 2.1 .1 & $\begin{array}{l}\text { Quel est l'impact des coûts d'agence et de l'information asymétrique sur le coût } \\
\text { de la dette et la disponibilité des fonds sous forme d'emprunt pour les PME? }\end{array}$ & 3 \\
\hline 2.1.2 & $\begin{array}{l}\text { Est-ce que les PME souffrent de discrimination de la part des institutions } \\
\text { financières en ce qui a trait au coût de la dette et à la disponibilité des fonds? }\end{array}$ & 5 \\
\hline 2.1.3 & $\begin{array}{l}\text { Sachant que les PME utilisent davantage le mode de financement par dette, } \\
\text { quels sont les déterminants de cette orientation? }\end{array}$ & \\
\hline 2.1.4 & $\begin{array}{l}\text { Est-ce que les coûts d'agence sont relativement plus importants } \\
\text { dans les PME que dans les plus grandes entreprises? }\end{array}$ & \\
\hline 2.1.5 & Quelles sont les caractéristiques des contrats d'emprunt des PME? & \\
\hline 2.2 & L'accès au financement par actions & 5 \\
\hline 2.2 .1 & $\begin{array}{l}\text { Existe-t-il un lien entre le niveau d'asymétrie de l'information } \\
\text { et le niveau de financement par actions utilisé par les PME? }\end{array}$ & \\
\hline 2.2.2 & $\begin{array}{l}\text { Quelles sont les caractéristiques des PME qui obtiennent du financement } \\
\text { par actions sur les marchés privés incluant les firmes de capital de risque? }\end{array}$ & 1 \\
\hline 2.2 .3 & Quelles sont les caractéristiques de propriété et de contrôle des PME? & \\
\hline 2.2 .4 & $\begin{array}{l}\text { Quels sont les problèmes d'agence des PME qui ont des propriétaires } \\
\text { multiples et de quelle façon ces problèmes sont-ils réglés? }\end{array}$ & \\
\hline 2.3 & La structure de capital optimale & 1 \\
\hline 2.3 .1 & $\begin{array}{l}\text { Quel est le niveau optimal d'emprunt pour les PME } \\
\text { et quelles en sont les bases théoriques? }\end{array}$ & 6 \\
\hline 2.4 & La politique de dividende & \\
\hline 2.4 .1 & $\begin{array}{l}\text { Quelles sont les causes exactes du niveau de dividendes } \\
\text { moins élevé dans les PME? }\end{array}$ & \\
\hline 2.4 .2 & $\begin{array}{l}\text { Est-ce que la théorie d'effet d'annonce relative aux dividendes } \\
\text { est applicable aux PME? }\end{array}$ & \\
\hline 2.5 & La forme de l'organisation & 1 \\
\hline \multirow[t]{2}{*}{ 2.5.1 } & $\begin{array}{l}\text { Quel est l'impact de la forme d'organisation adoptée } \\
\text { sur les coûts de financement des PME? }\end{array}$ & \\
\hline & Total & 55 \\
\hline
\end{tabular}

Revue internationale P.M.E., vol. 14, nº 1, 2001 


\section{Bibliographie}

BARTON, S.L. et C.H. MAtTeWs (1989), «Small firm financing: implications from a strategic management perspective », Journal of Small Business Management, vol. 27, $\mathrm{n}^{\mathrm{o}} 1$, p. 1-7.

BINKS, M.R., C.T. ENNEW et G.V. ReED (1992), «Information asymmetries and the provision of finance to small firms », International Small Business Journal, vol. 11, $\mathrm{n}^{\mathrm{o}} 1$, p. 35-46.

BROPHY, D.J. et J.M. SHULMAN (1992), «A finance perspective on entrepreneurship research », Entrepreneurship Theory and Practice, vol. 16, no 3, p. 61-71.

Chaganti, R., D. Decarolis et D. DeEds (1995), « Predictors of capital structure in small ventures », Entrepreneurship Theory and Practice, vol. 20, nº 2, p. 7-18.

Colot, V. et P.A. Michel (1996), «Vers une théorie financière adaptée aux PME. Réflexion sur une science en genèse ", Revue internationale PME, vol. 9, n 1, p. 143-166.

Cossette, P. (1995), La cognition comme objet d'étude dans la littérature scientifique sur la PME et l' entrepreneuriat, $\mathrm{II}^{\mathrm{e}}$ Congrès international francophone de la PME, Paris, p. 487-524.

CossetTe, P. (1997), «Les travaux publiés dans la Revue internationale PME depuis sa fondation : caractéristiques et tendances », Revue internationale PME, vol. 10, $\mathrm{n}^{\mathrm{0}} 2$, p. $109-128$.

HUSTEDDE, R.J. et G.C. PULVER (1992), «Factors affecting equity capital acquisition : the demand side », Journal of Business Venturing, vol. 7, n ${ }^{\circ}$, p. 363-374.

LOW, M. et I.C. MACMILLAN (1988), «Entrepreneurship : past research and future challenges », Journal of Management, vol. 14, $\mathrm{n}^{\circ}$ 2, p. 139-161.

MACMiLLAN, I.C. (1993), « The emerging forum for entrepreneurship scholars », Journal of Business Venturing, vol. 8, $\mathrm{n}^{\circ}$ 5, p. 377-381.

MCMAHON, R.G.P. et A.M.J. STANGER (1995), «Understanding the small enterprise financial objective function », Entrepreneurship Theory and Practice, vol. 19, n 4, p. 21-39.

MERIKAS, A., G.D. BRUTON et G.S. VOZIKIS (1993), «The theoretical relationship between the strategic objective of sales growth and the financial policy of the entrepreneurial firm », International Small Business Journal, vol. 11, n 3, p. 59-67.

Norton, E. (1991), «Capital structure and small public firms », Journal of Business Venturing, vol. 6, $\mathrm{n}^{\circ}$ 4, p. 287-303.

PETTIT, R. et R.F. SINGER (1985), « Small business finance : a research agenda », Financial Management, vol. 14, $\mathrm{n}^{\mathrm{o}}$ 3, p. 47-60.

WORTMAN, M.S.J. (1987), «Entrepreneurship : an integrating typology and evaluation of the empirical research in the field », Journal of Management, vol. 13, $n^{\circ}$ 2, p. 257-279.

Revue internationale P.M.E., vol. 14, n 1, 2001 